\title{
Upgrade of the ATLAS detectors and trigger for the High Luminosity LHC: tracking and timing for pile-up suppression
}

\author{
M. Testa ${ }^{\mathrm{a}, *}$ \\ ${ }^{a}$ INFN LNF, Frascati(RM), Italy
}

\begin{abstract}
The High Luminosity-Large Hadron Collider is expected to start data-taking in 2026 and to provide an integrated luminosity of $3000 \mathrm{fb}^{-1}$, giving a factor 10 more data than will be collected by 2023 . This high statistics will make it possible to perform precise measurements in the Higgs sector and improve searches of new physics at the TeV scale. The peak luminosity is expected to be $7.5 \times 10^{34} \mathrm{~cm}^{-2} \mathrm{~s}^{-1}$, corresponding to about 200 proton-proton interactions per event (pile-up), which will increase the rates at each level of the trigger and degrade the reconstruction performance. To cope with such a harsh environment many sub-detectors of the ATLAS experiment will be upgraded and some completely substituted. The Trigger-DAQ system will be upgraded. In this talk an overview of two new sub-detectors enabling powerful pile-up suppression, a new Inner Tracker and a High Granularity Timing Detector, will be given, describing the two technologies, their performance, and their interplay. Emphasis will also be given to the possibility of using tracking and timing information at the earliest, hardware based, ATLAS trigger stage.
\end{abstract}

\section{Introduction}

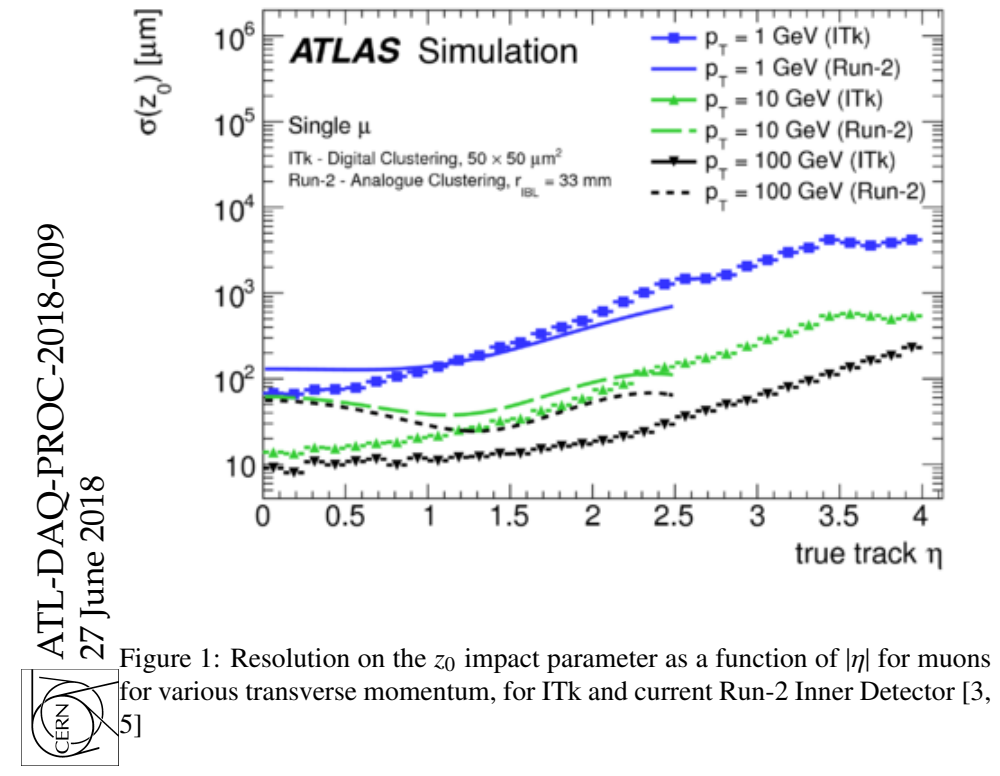

The HL-LHC will deliver an integrated luminosity of 3000 $\mathrm{fb}^{-1}$ in ten years starting from 2026, to perform precise measurements in the Higgs sector and improve searches for new physics at the $\mathrm{TeV}$ scale. About 200 pile-up collisions per bunch crossing $(\mu)$ are expected at the HL-LHC, to be compared with $\sim 40$ during Run-2. The ATLAS Phase-2 Scoping Document [1] provides an overview of the plans for this high luminosity phase. The current Inner Detector (ID) of the
ATLAS experiment [2] will be replaced by a new all-silicon tracker (ITk) [3, 4] to maintain tracking performance in this high-occupancy environment and to cope with the increase of approximately a factor of ten in the integrated radiation dose. The ITk will have a larger angular coverage than the ID, up to $|\eta|<4$, to efficiently mitigate pile-up effects, especially in the jet reconstruction and to provide a better reconstruction of forward leptons. The ITk will have a better impact parameter resolution with respect to the ID due to reduced material budget. In particular, the improvement in the resolution of $z_{0}$ impact parameter of tracks, shown in Figure 1, is crucial to assign correctly tracks to the primary vertex. The deterioration at large $|\eta|$ is due to increased material crossed and larger distance from the first detector layer to the collision point. A proposed upgrade for HL-LHC is the High Granularity Time Device (HGTD), with thickness $6 \mathrm{~cm}$, located in front of endcap calorimeter at $2.4<|\eta|<4$. The HGTD is equipped with Low Gain Avalanche Diodes Cell with size $1.3 \times 1.3 \mathrm{~mm}^{2}$, providing a timing resolution of $\sim 30 \mathrm{ps}$. A first design was given in [1]. The HGTD technical proposal is in preparation. The motivation for this upgrade is to use timing information to improve the pile-up suppression in the online and offline reconstruction. At $\mu=200$, 5-7 vertices within tracker resolution at large $|\eta|$ are expected to be merged, due to poor $z_{0}$ resolution. Degraded performance of pile-up suppression techniques and isolation efficiency at large $|\eta|$ are therefore expected. Timing information may help pile-up rejection resolving nearby vertices. Timing information could also improve trigger capabilities at $40 \mathrm{MHz}$, where the tracking information is not yet available, but is not considered in current baseline.

${ }^{*}$ Corresponding author, on behalf of the ATLAS Collaboration
Email address: marianna. testa@lnf.infn. it (M. Testa) 


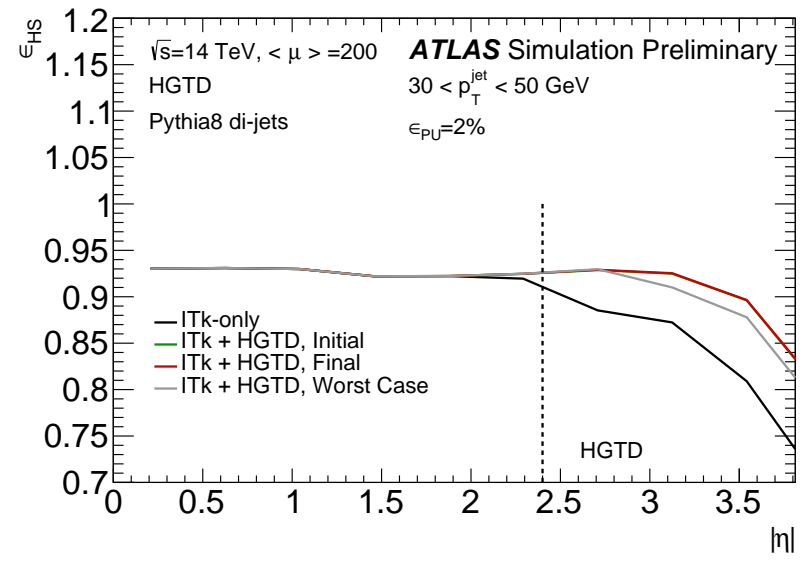

Figure 2: The efficiency to select hard-scatter jets with $30<p_{T}<50 \mathrm{GeV}$, as a function of $|\eta|$ for a $2 \%$ pile-up jets efficiency with and without HGTD [8]. The green line is very close to the red line. See text for details.

\section{Pile-up jet suppressions with ITk and HGTD}

The impact of ITk and HGTD to improve pile-up jet rejection have been addressed using a track-based pile-up jet tagging algorithm. This class of algorithms, which exploits the pointing of tracks to the production vertex $[6,7]$, is powerful to tag pile-up jets but is sensitive to the $z_{0}$ resolution of the tracks in the jets. Figure 2 shows the efficiency for hard-scatter jets versus $\eta$ for jets with $30<p_{T}<50 \mathrm{GeV}$ for a $2 \%$ pile-up jets efficiency, for several scenarios related to different timing resolutions. The "Initial" HGTD scenario corresponds to the expected performance at the beginning of the HL-LHC operation. The "Final" scenario represents the expected timing resolution at the end of the HL-LHC program assuming that the sensors and readout electronics at $R<320 \mathrm{~mm}$ are replaced after half of the integrated luminosity has been delivered. The "Worst Case" scenario assumes a timing resolution of 60 ps per hit for the sensors. The HGTD recovers the 10-30\% drop in efficiency observed in the forward region, allowing to maintain similar pile-up jet suppression performance as in the central region.

\section{Tracking for trigger at HL-LHC}

The Trigger and Data Acquisition (TDAQ) system will be upgraded for the HL-LHC operations [9]. In particular, the upgraded trigger system will perform hardware-based tracking. The baseline architecture design is based on a single Level- 0 (L0) hardware trigger with a detector readout rate of $1 \mathrm{MHz}$ and a maximum latency of $10 \mu \mathrm{s}$. Tracking information is used both for a regional and global Hardware Tracking for the Trigger (rHTT, gHTT). The rHTT searches for all tracks with $p_{\mathrm{T}}>2$ $\mathrm{GeV}$ in limited regions around L0 trigger objects. The rHTT will use only $10 \%$ of ITk hits from the strip system and outer pixel layers. Regional tracking will accomplish a significant reduction in the L0 output rate from $1 \mathrm{MHz}$ to $400 \mathrm{kHz}$. In Fig. 3 the resulting rate for a four-jet trigger after requiring a common vertex versus the fourth jet $p_{\mathrm{T}}$ threshold is shown. The gHTT searches for all tracks with $p_{\mathrm{T}}>1 \mathrm{GeV}$ at a nominal rate of 100

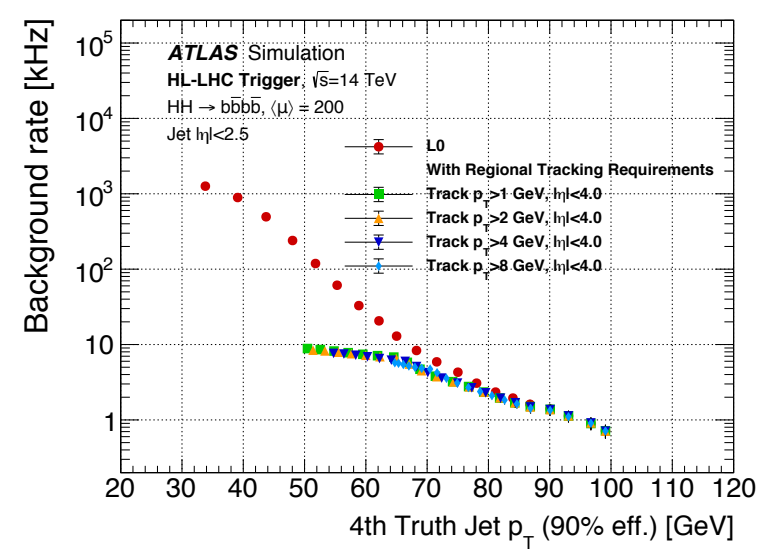

Figure 3: Rate for a four-jet trigger requiring the jets to be consistent with a common vertex using rHTT [9]. The rate is shown as a function of the effective offline threshold for the lowest ("4th") $\mathrm{p}_{\mathrm{T}}$ jet.

$\mathrm{kHz}$ and provides high-quality tracks for lepton isolation, primary vertex reconstruction, b-tagging, and missing transverse energy reconstruction. An evolved scenario with a two-level hardware trigger design, L0 and Level-1 (L1), is also considered as a mitigation strategy in case pile-up conditions at the HL-LHC either challenge the readout capabilities of certain detectors to the limits of the available bandwidth, or in case the rates of hadronic trigger signatures are much larger than the current predictions. The L0 trigger rate is up to $4(2) \mathrm{MHz}$ with $10 \mu$ s latency, followed by a L1 trigger rate of $600(800) \mathrm{kHz}$ and a latency up to $35 \mu \mathrm{s}$. In this design, a hardware-based track reconstruction is implemented in the L1 trigger system; these reconstructed tracks are combined with calorimeter- and muonbased trigger objects to form the L1 decision. The usage of regional tracking information in the earliest trigger levels will enable a new VBF Higgs inclusive trigger, reducing the L0 rate from $33 \mathrm{kHz}$ to $5 \mathrm{kHz}$ with a signal acceptance of $6.6 \%$ in the baseline scenario and up to $10 \%$ in the evolved scenario. More information can be found in [9].

[1] ATLAS Collaboration, "ATLAS Phase-II Upgrade Scoping Document", CERN-LHCC-2015-020, https://cds . cern. ch/record/2055248

[2] ATLAS Collaboration, "The ATLAS Experiment at the CERN Large Hadron Collider", JINST 3 (2008) S08003

[3] ATLAS Collaboration, "Technical Design Report for the ATLAS Inner Tracker Pixel Detector', in preparation

[4] ATLAS Collaboration, "Technical Design Report for the ATLAS Inner Tracker Strip Detector", CERN-LHCC-2017-005, https://cds.cern.ch/record/2257755

[5] ATLAS Collaboration, "Plots from Technical Design Report for the ATLAS Inner Tracker Pixel Detector", https://atlas.web.cern.ch/Atlas/GROUPS/PHYSICS/PLOTS/ITK-2018001

[6] ATLAS Collaboration, "Tagging and suppression of pileup jets with the ATLAS detector", ATLAS-CONF-2014-018, https://cds.cern.ch/record/1700870

[7] ATLAS Collaboration, "Forward Jet Vertex Tagging: A new technique for the identification and rejection of forward pileup jets", ATL-PHYSPUB-2015-034, https://cds . cern. ch/record/2042098

[8] ATLAS Collaboration, "Technical Proposal: A High-Granularity Timing Detector for the ATLAS Phase-II Upgrade", in preparation

[9] ATLAS Coll., "Technical Design Report for the Phase-II Upgrade of the ATLAS Trigger and Data Acquisition System", in preparation 